\title{
Real-Time Estimation of Drivable Image Area based on Monocular Vision
}

\author{
A. Miranda Neto, A. Corrêa Victorino, I. Fantoni and J. V. Ferreira
}

\begin{abstract}
Camera-based estimation of drivable image areas is still in evolution. These systems have been developed for improved safety and convenience, without the need to adapt itself to the environment. Machine Vision is an important tool to identify the region that includes the road in images. Road detection is the major task of autonomous vehicle guidance. In this way, this work proposes a drivable region detection algorithm that generates the region of interest from a dynamic threshold search method and from a drag process (DP). Applying the DP to estimation of drivable image areas has not been done yet, making the concept unique. Our system was has been evaluated from real data obtained by intelligent platforms and tested in different types of image texture, which include occlusion case, obstacle detection and reactive navigation.
\end{abstract}

\section{INTRODUCTION}

$\mathrm{T}$ HE perception of the environment is a major issue in autonomous and (semi)-autonomous systems. In the last three decades, visual navigation for mobile robots has become a source of countless research contributions [1]. The development of autonomous or driverless vehicles capable of moving on unknown and dynamic environments could provide important benefits to: patrolling, search and rescue, outdoor and indoor building inspection, real-time monitoring, autonomous cruise control, collision avoidance system and pre-crash system, intelligent speed adaptation, etc [1], [2].

However, the challenge to construct robust methods, and, in most cases, optimized systems, remains an open problem. Some of these applications include: the Grand Challenge [3]; Urban Challenge [4]; Advanced Driver Assistance Systems (ADAS) [5]; autonomous perception system [6], [7]. These applications have a common issue: providing to the robot/vehicle platform the capability of perceiving and interacting with its neighbour environment.

In this way, perception vision systems have been designed to investigate the information's road, and different techniques on automatic and semi-automatic road extraction methods using monocular vision were proposed in the literature [6], [8], [9], [10], [11], [12]. The ability to identify the region that includes the road in images captured using vehicle mounted cameras is a useful function [13]. Determining the area of free road ahead is a key component

Manuscript received April 11, 2013.

Arthur de Miranda Neto and Janito Vaqueiro Ferreira are with the Autonomous Mobility Laboratory (LMA) at FEM/UNICAMP, Brazil.

Alessandro Corrêa Victorino and Isabelle Fantoni are with the Heudiasyc laboratory UMR 7253 CNRS/UTC, France. of several driving assistance modules [10]. Monocular camera systems are preferred to stereo camera systems because monocular systems have advantages in terms of reduced costs and the facility with which they can be fitted to vehicles [13]. The monocular vision contribution to the DARPA Grand Challenge [9] shows that the range of lasers was approximately 22 meters, whereas the monocular vision module often looks 70 meters ahead.

In this way, we propose a machine vision algorithm capable of identifying the free-navigable area (region of interest). Our proposed monocular system was tested in different types of road surfaces, which include occlusion case, obstacle detection and reactive navigation.

The paper is organized as follows. In Section II we present the sensor perception review and road detection contributions. Our proposed method is presented in Section III. From the Section IV the results are presented and the conclusions are given in Section V.

\section{RELATED WORKS}

The perception layer, on-boarded in intelligent transportation systems, uses many types of sensors, including ultrasonic sensors, laser rangefinders, radar, cameras, etc. However, these sensors are not perfect: ultrasonic sensors are cheap but suffer from specular reflections, and laser rangefinders and radar provide better resolution but are more complex and more expensive [6]

According to [8], the vision-based sensors are defined as passive sensors and it can be used for some specific applications: road marking localization, traffic signs recognition and obstacle identification. However, vision sensors are less robust than millimeter-wave radars in foggy, night, or in direct sun-shine conditions. On the other hand, range-based obstacle detection systems have difficulty for detecting small or flat objects on the ground, and range sensors are also unable to distinguish between different types of ground surfaces [6]. Notwithstanding, the main problem with the use of active sensors is represented by interference among sensors of the same type, hence, foreseeing a massive and widespread use of these sensing agents, the use of passive sensors obtains key advantages [8].

Additionally, when incorporating several types of sensors, there is an increase of autonomy and "intelligence" degrees, especially in relation to navigation in unknown environments. In contrast, the type and quantity of sensors determine the volume of data for processing that requires, in 
most cases, a high computational cost. For unstructured environments, the scenario for study is dynamic, with several elements in motion. Thus, running an autonomous or semiautonomous system involves carrying out complex, and nondeterministic operations in real time.

To investigate the information's road, different techniques on automatic and semi-automatic road extraction methods are proposed in the literature: for a system trained by driving a robot through its environment, a vision-based obstacle detection allows to classify each individual image pixel as either an obstacle or a ground based on its color appearance [6]; for robot navigation in agricultural environments or hazardous related areas, a method for extracting and tracking man-made roads segments color images in small areas was proposed. These small areas are characterized later by color and texture attributes, and features are classified using the $\mathrm{K}$ NN rule or the Support Vector Machines method [8]; a method for identifying drivable surfaces in difficult unpaved and off-road terrain conditions was proposed based on computer vision and lidar. Computer vision then construct appearance models to find drivable surface outward into the far range [9]; from a previously extracted road segments (manually or semi-automatically) in a traffic-free reference video record on a first drive, a road-detection method to infer the areas of the image depicting road surfaces without performing any image segmentation was presented by [10]. It uses a dynamic background subtraction based on Otsu thresholding algorithm; a partition-based algorithm for classification of outdoor terrains using monocular camera was proposed by [11]. This work was based on learning from the data by fruitfully exploiting the data obtained on the fly; a vision-based road detection method was proposed by [12] to realize visual guiding navigation for autonomous land vehicles. In this case, the images are segmented into road and non-road region by using Otsu thresholding algorithm, which included the Monte Carlo method with the road boundary extraction based on Canny.

Aware that in the majority of the navigation systems, the machine vision system is working together with other sensors, added to its low cost, we present a monocular vision-based system that includes a robust road detection algorithm. Because it uses simple techniques and fast algorithms, the system is capable to present a good performance, where the commitment between processing time and images acquisition is fundamental.

Furthermore, although extremely complex and highly demanding, thanks to the great deal of information it can deliver, the machine vision is a powerful means for sensing the environment and has been widely employed to deal with a large number of tasks in the automotive field [15]. However, complex machine-vision systems can lead to some losses due to the processing time. Thinking about the existing relation between a real-time decision system and a camera acquisition system that operates in a specific acquisition rate, the decision for a more complex machine vision system possibly leads to an excessively slow system for an independent real-time application. The great amount of information would not necessarily lead to better decisions and could also harm the performance of the system, overloading it.

With respect to these previous works, the road detection method proposed in this article presents a major contribution related to the performance and efficiency computation, responding to the real-time requirements. Then, taking into account that it has been estimated that humans perceive visually about $90 \%$ of the environment information required for driving [15], it is not a bad idea to reduce information acquired by a vision system, in order to reduce processing time. In that case, based on the idea to reduce information acquired and in order to reduce processing time, the road detection method proposed here is based on an automatic image discarding criteria [16], which is based on Pearson's Correlation Coefficient (PCC), and on a low complexity and easy implemented solution. It improves the performance of a real-time system by choosing, in an automatic way, which images should be discarded and which ones should be treated at the visual perception system. Additionally, in most real-world systems, there is little knowledge of future input events and Dynamic Power Management (DPM) decisions have to be taken based on uncertain predictions. Thus, according to [17], the rationale in all predictive techniques is that of exploiting the correlation between the past history of the workload and its near future in order to make reliable predictions about future events. Moreover, workload observation and prediction should not consume significant energy. Therefore, taking into account the temporal coherence between consecutive frames, we have also proposed a new DPM methodology applied to a robotic visual machine perception, which includes a Cumulative Impact Data Management [18].

Finally, in order to decrease the volume of data for processing, some systems have been designed to investigate only a small portion of the road ahead of the vehicle where the absence of other vehicles can be assumed [15]. Otherwise, the sky region is not a region of interest, and the horizon line threshold is applied to generate a road image [19]. Stanford Racing Team [9] implemented the horizon finding algorithm originally proposed by [20] to eliminate all pixels above that horizon. In this way, to compose our road detection method, we have proposed a robust horizon finding algorithm that finds the horizon line and apply it to generate the free-navigable area (region of interest) [21].

\section{DrivaBle IMAGE AREA}

Many works have focused upon the obstacle avoidance problem. In a general way, the primary purpose is to detect the free space immediately around the mobile robot. These algorithms carry through operations on images, with the purpose to reduce noise and to segment them.

One way to perform segmentation of an image is to use 
thresholds. This type of segmentation technique, called thresholding, is very simple and computationally fast, however the identification of the ideal threshold can be sufficiently complicated. The best thing to do in this case is to use techniques and algorithms that search the thresholds automatically. Thresholding methods are divided in two groups: global and local. The global ones divide the image using only one threshold and the local ones are those that divide the image in sub-images and for each one of them a threshold is defined [22].

We propose a global thresholding method, which seeks not the ideal threshold for the whole image, but an ideal threshold associated with the region of interest, i.e. navigable area. In this case, we apply the sky removal method proposed in [21], because once using a global segmentation method, not always the analysis of a bigger image portion can contribute for a better result in the most critical region (region closer to the vehicle) where obstacles should be detected and avoided as fast as possible. On the contrary, when discarding the superior portion of the original image, sky removal, we are capable to get a more efficient segmentation and to distinguish with higher precision the obstacles from the navigable area. Moreover, for land vehicle navigation, Fig. 2 illustrates that probably for images that possess the horizon (sky) in its composition, the algorithm may not have a satisfactory result. On the other hand, in our methodology, after sky removal, the new result can be seen in Fig. 2 (c).

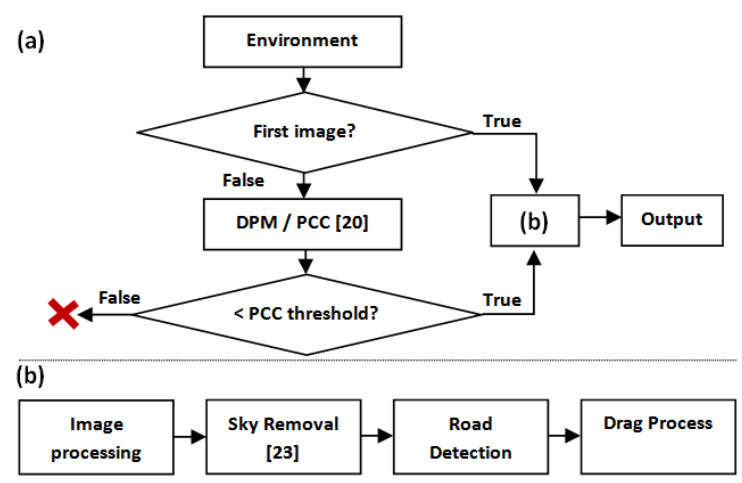

Fig. 1 - Block diagram of our system.
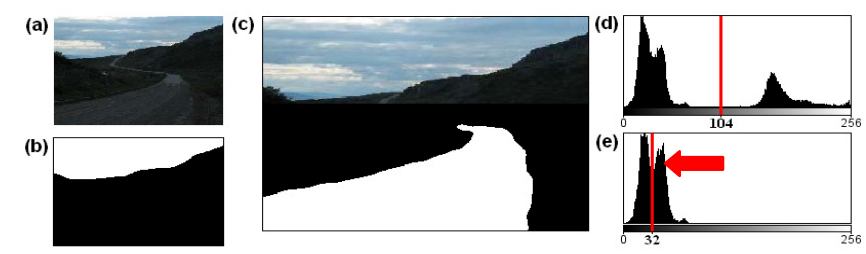

Fig. 2 - (a) Original image; (b) Free-area before sky removal; (c) Free-area after sky removal [21]; (d) Otsu threshold (104) to (a); Otsu threshold (32) to (c).

\section{A. Image pre-processing}

Most research groups face this problem using highly sophisticated image filtering algorithms [15]. In this work we use a color or gray-level image and smooth them using a
Gaussian filter. The Gaussian smoothing operator is a 2-D convolution operator. It acts as low-pass frequency filters [23]. In order to reduce the number of data, it includes the resolution reduction of image (to $96 \times 72$ ).

For find the drivable surfaces, [3] projects drivable area from the laser scan analysis into the camera image. This quadrilateral area is between 10 and 20 meters ahead of the robot. In this real-time approach, the basic idea is to consider a given region in the actual image as drivable. It assumes that the bottom center of the image contains road pixels for a large majority of the time [27]. This technique was first presented by [6].

In our proposed method, if the image is colored, in order to utilize the most important information of the color image, the candidate color channel that was dominant in the bottom center of the image is selected to generate the histogram image [12], Fig. 3 (a): yellow region. It is described by Eq. (1).

$$
C c=\underset{\{R, G, B\}}{\arg \max }\left(N_{R}, N_{G}, N_{B}\right)
$$

where $C c$ means the dominant color channel.

\section{B. Image segmentation based on Otsu method}

In this work, the purpose of segmentation is the road detection. Right after the images pre-processing, we can start searching for an ideal threshold based on Otsu [14].

The main characteristic of Otsu method is the maximization of the intra-classes variance of the image. The thresholding process is seen as the partitioning of pixels of an image in two classes: C1 (object) and C2 (background). This method is recursive and searches the maximization for the cases: $\mathrm{C} 1=\{0,1, \ldots, \mathrm{T}\}$ and $\mathrm{C} 2=\{\mathrm{T}+1, \mathrm{~T}+2, \ldots, \mathrm{N}-1\}$, where $\mathrm{T}$ is the chosen threshold and $\mathrm{N}$ the number of intensity levels of the image. This method searches exhaustively for the threshold that minimizes the intraclasses variance, defined as a weighted sum of variances of the two classes, it is described by Eq. (2).

$$
\sigma_{W}^{2}(T)=q_{1}(T) \sigma_{1}^{2}(T)+q_{2}(T) \sigma_{2}^{2}(T)
$$

where $\sigma_{W}^{2}(T)$ is the intra-class variance, $q_{1}(T)$ is the proportion of background pixels, $\sigma_{1}^{2}(T)$ is the intensity variance of background pixels, $q_{2}(T)$ is the proportion of foreground pixels and $\sigma_{2}^{2}(T)$ is the intensity variance of foreground pixels. The class probabilities are estimated as:

$$
q_{1}(T)=\sum_{i=1}^{T} H(i) \text { and } q_{2}(T)=\sum_{i=T+1}^{N} H(i)
$$

The class means are given by:

$$
\mu_{1}(T)=\sum_{i=1}^{T} \frac{i H(i)}{q_{1}(T)} \text { and } \mu_{2}(T)=\sum_{i=T+1}^{N} \frac{i H(i)}{q_{2}(T)}
$$


Finally, the individual class variances are:

$$
\begin{aligned}
& \sigma_{1}^{2}(T)=\sum_{i=1}^{T}\left[i-\mu_{1}(T)\right]^{2} \frac{H(i)}{q_{1}(T)} \\
& \sigma_{2}^{2}(T)=\sum_{i=T+1}^{N}\left[i-\mu_{2}(T)\right]^{2} \frac{H(i)}{q_{2}(T)}
\end{aligned}
$$

where $H$ is the histogram of the selected channel in Eq. (1).

One of the great advantages of this method is that it does not restrict itself to the type of histogram of the image, that is, it can be applied to unimodal, bimodal or multimodal histograms, but it presents better performance in images with bigger intensity variance. Its main disadvantage is its sensitivity to noise in the image, what can be reduced with the application of a filter.

Region recognition can be handled by popular thresholding algorithm such as Maximum Entropy, Invariant Moment and Otsu thresholding method (OTM). For road detection, because OTM supplies a more satisfactory performance in image segmentation, it was used to overcome the negative impacts caused by environmental variation [12].

Furthermore, some authors consider the OTM as one of the best choices for real-time applications in machine vision [24], [25]. It still remains one of the most referenced thresholding methods [26].

\section{Estimation of Drivable Image Area}

From the image processing and sky removal steps, in order to obtain a multimodal $2 \mathrm{D}$ drivability free-area, $F A_{w}$, i.e. free-navigable area detection, the algorithm performs the following tasks:

1. Task 1: Due to the different image textures on different roads, the original image, Figure 3 (a), and its negated, Fig. 3 (c), are submitted to the Otsu's method in Eq. (2). The algorithm then selects an image with the highest percentage of navigable area (white points) in the bottom center of the image, as shown in Fig. 3 (a): yellow area. The original image and its Otsu's result, $\mathrm{Os}^{+}$, can be seen in the Fig. 3 (a) and (b). The negated image and its Otsu's result, $O s^{-}$, can be seen in the Fig. 3 (c) and (d).

2. Task 2: Whereas there are homogeneous regions in the image, and in order to identify the limits of the road (which includes the obstacles), the Canny edge detector [28] was employed as input of Hough transform [29] due to its robust performance and accurate edge localization. Respectively, the results can be seen in the Fig. 3 (e) and (f). Then, from the bottom center of the image, the algorithm concludes by finding a single image mass, $H c$, Fig. 3 (g). It may also help to identify the textureless regions classified as road region, specular surfaces, traffic markings, etc.
A multimodal road image is then triggered based on the weighted average of the images intensities. It is described in Eq. (7).

$$
F A_{w(x, y)}=\frac{F A_{w(x, y)}+O s_{(x, y)}+H c_{(x, y)}}{3}
$$

where $F A_{w(x, y)}$ (left) is the intensity of the $\mathrm{i}^{\text {th }}$ pixel after update in the new image $F A_{w}, F A_{w(x, y)}$ (right) is the intensity of the $\mathrm{i}^{\text {th }}$ pixel in the old image $F A_{w(x, y)}, O s_{(x, y)}$ is the intensity of the $i^{\text {th }}$ pixel in image obtained in Task 1 , $H c_{(x, y)}$ is the intensity of the $\mathrm{i}^{\text {th }}$ pixel in image obtained in Task 2.

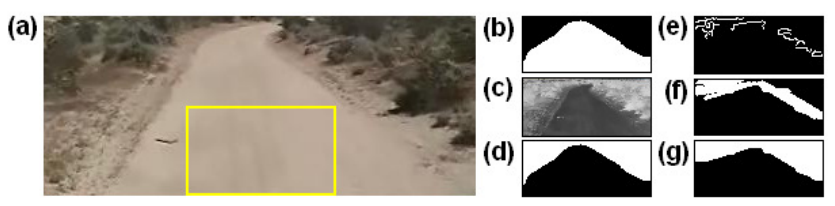

Fig. 3 - (a) Original image after sky removal and its Otsu's result in (b); (c) negated image and its Otsu's result in (d); (e) Canny edge detection result; (f) Hough transform result; (g) a single mass in the image (f).

Due the small variance of shades between objects, false path-markings, false route-markings, shadows, etc, the next step is applied to re-project this 2D drivability free-navigable area by considering a drag process presented in the next section.

\section{Drag forces: process}

In fluid dynamics, drag forces act in a direction opposite to the oncoming flow velocity, i.e. forces (or resistance) that oppose the relative motion of an object through a fluid [30]. Coming again to the domain of image, our objective is to classify the road image in terms of fluidity and navigability. In this case, the obstacles represent the forces that oppose the relative motion of a robot through a route. A new multimodal 2D drivability free-area by considering the drag process is described in Eq. (8):

$$
F A_{d\left(x, y_{i}\right)}=F A_{w\left(x, y_{i}\right)}-\left(F A_{w\left(x, y_{i}\right)} *\left(1-\frac{F v}{D f}\right)\right)
$$

where for each image column $x$ : (1) the algorithm initiates by analyzing each image row $y$, from bottom to top; (2) the drag forces, $D f$, and flow velocities, $F v$, are initialized with a value by default equal to $h * 255$, where $h$ is the image height and 255 the highest intensity level of a pixel. Then, for $y=h-1$ to $y=0$, the $D f$ and $F v$ values are updated by:

$$
\begin{gathered}
D f=D f-255 \\
F v=(F v-255)-\left(255-F A_{w\left(x, y_{i}\right)}\right)
\end{gathered}
$$


where $F A_{d\left(x, y_{i}\right)}$ is the intensity of the $\mathrm{i}^{\text {th }}$ pixel in the image. If $F v<=0$ then $F v=0$. The velocity, reference area and drag coefficient attributes are equal to 1 .

Right after applying the equations Eq. (7) to Eq. (10), the final result to Fig. 3(a) is shown in Fig. 4 (b). Additionally, a hypothetical multimodal $2 \mathrm{D}$ drivability road image $5 \times 5$ is presented in Fig.5 as an example of this drag process.
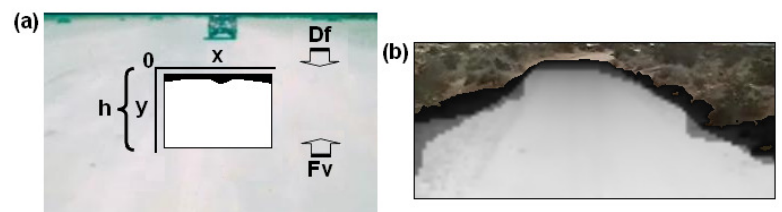

Fig. 4 - (a) The drag process; (b) The final result to Fig. 3(a) is a new multimodal $2 \mathrm{D}$ drivability free-navigable by considering the drag process: pixel intensities $F A_{d\left(x, y_{i}\right)}>=10$.

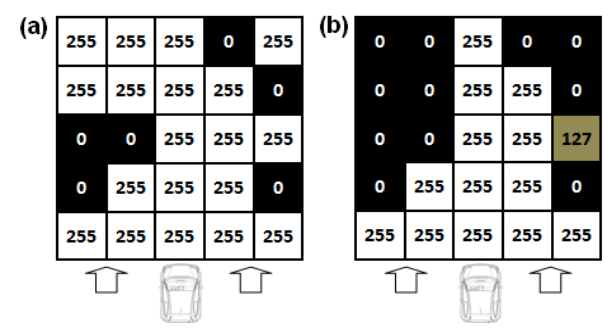

Fig. 5 - (a) Hypothetical multimodal 2D drivability road image 5x5; (b) Right after apply the Eq. (8), the new result by considering the drag process.

\section{EXPERIMENTAL RESULTS}

As has been shown above in Section III.C, the Fig. 4 (a) and (b) show the application of our proposed method in desert context [31]. In this section, we present results on real, dynamic and unknown environments. These results were obtained using two experimental vehicles. As shown in Fig. 9 (a) and (b), both vehicles are equipped with a camera to acquire color images and a CAN-bus gateway to provide the speed of the rear-wheels.

At first stage of testing, in order to evaluate the proposed algorithm performance, we used an urban and real experimental test-bank. These data from Fig. 6 to Fig. 7 were obtained using the vehicle shown in Fig. 9 (a). A typical urban environment was selected and its results are presented in Fig. 6. All false-navigable areas (red) in Fig. 6 (c) are eliminated by applying the drag process resulting in Fig. 6 (d). Additionally, different types of image texture (road surfaces) were selected and its results are presented in Fig. 7 (a) to (f). A result for a shadow context is presented in Fig. 7 (d). An occlusion case (vehicle) is shown in Fig. 7 (b).

At second stage of testing, in order to evaluate the proposed algorithm performance to autonomous displacement, the experiments on real-time conditions were performed using the vehicle VERO shown in Fig. 9 (b). In Fig. 8 (a), (b) and (c) show the successful task execution to go through a gate in off-road context. Fig. 8 (d) and (e) present the obstacle detection and an open-loop reactive navigation [32]. In all experiments there was no collision. Additionally, different type of image texture (road surfaces) was also selected and its result is presented in Fig. 8 (f).

The computational mean time of the road detection process was about $10 \mathrm{~ms}$. It was tested on a $2.5 \mathrm{GHz}$ Intel Core 2 Quad processor, Microsoft Windows XP Professional SP3. This time includes the resolution reduction of image and Gaussian filtering. The resolution reduction did not change the results. For additional results see the video [33].

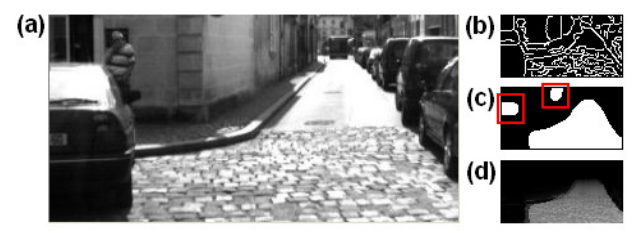

Fig. 6 - (a) Original image after sky removal and its Otsu's result in (c); (b) Canny edge detection result; (d) A multimodal 2D drivability free-area by considering the drag process.

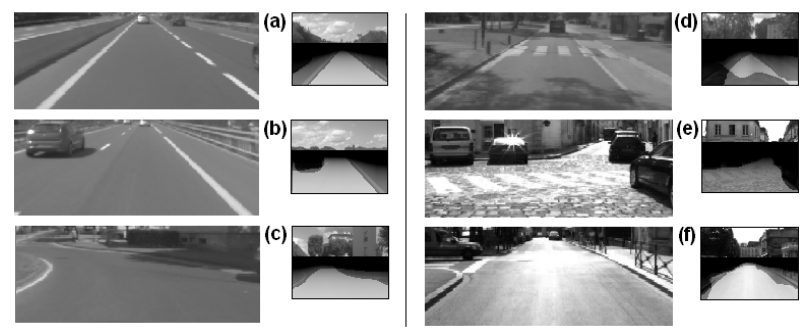

Fig. 7 - Urban and real experimental test-bank.

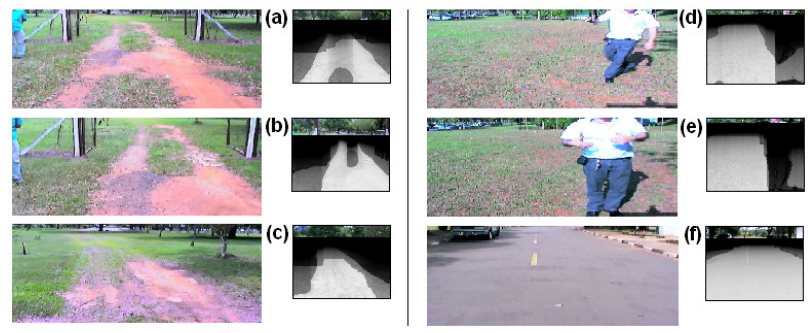

Fig. 8 - Real-time autonomous displacement.

\section{CONCLUSION}

The challenge to construct robust methods of image processing and analysis is far from being achieved. In this work, we have proposed a machine vision algorithm capable of identifying the free-navigable area from images captured by a single camera. It is important to notice that our algorithm is not based on previous knowledge of the environment neither camera calibration. Future work would be also focused to provide ground truth measurements from a front mounted radar and/or LIDAR system. 


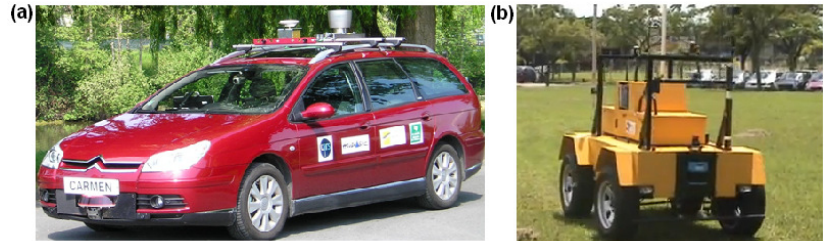

Fig. 9 - The experimental vehicles: (a) Carmen vehicle at Heudiasyc Laboratory in Compiègne, France; (b) Autonomous vehicle (VERO) at Renato Archer IT Center (CTI) in Campinas, Brazil.

\section{ACKNOWLEDGMENT}

The authors wish to thank Dr. Luiz Gustavo Bizarro Mirisola, Dr. Samuel S. Bueno, Dr. Josué Jr. G. Ramos, Mr. Hélio Azevedo and Mr. Gerald Dherbomez for their kind attention to our work, for the useful discussions and support in data acquisition.

\section{REFERENCES}

[1] F. Bonin-Font, A. Ortiz and G. Oliver, (2008), "Visual Navigation for Mobile Robots: A Survey", Journal of Intelligent and Robotic Systems. 53, 3, 263-296.

[2] S. A. Rodríguez Flórez, (2010), "Contributions by Vision Systems to Multi-sensor Object Localization and Tracking for Intelligent Vehicles", Thesis, UTC, France.

[3] S. Thrun, et al. (2006), "Stanley, the robot that won the DARPA Grand Challenge", Journal of Robotic Systems, Volume 23, Issue 9, 661-692, 2006, ISSN:0741-2223.

[4] Team Berlin (2007), "Spirit of Berlin: An Autonomous Car for the DARPA Urban Challenge Hardware and Software Architecture" [retrieved Jan 05, 2010]

[5] O. Gietelink, J. Ploeg, B. De Schutter, and M. Verhaegen, (2006) "Development of advanced driver assistance systems with vehicle hardware-in-the-loop simulations," Vehicle System Dynamics, vol. 44, no. 7.

[6] I. Ulrich and I. Nourbakhsh, (2000), "Appearance-Based Obstacle Detection with Monocular Color Vision", Proceedings of the AAAI National Conference on Artificial Intelligence, 866-871.

[7] B. Kim, P. Hubbard, D. Necsulescu, (2003), "Swarming Unmanned Aerial Vehicles: Concept Development and Experimentation, A State of the Art Review on Flight and Mission Control",Technical Memorandum.

[8] G. Aviña-Cervantes, M. Devy and A. Marín, (2003), "Lane Extraction and Tracking for Robot Navigation in Agricultural Applications", Proceedings of the IEEE ICAR 2003.

[9] H. Dahlkamp et al., (2006), "Self-Supervised Monocular Road Detection in Desert Terrain". Proceedings of the Robotics Science and Systems Conference.

[10] F. Diego, J. M. Álvarez, J. Serrat and A. M. López, (2010), "Vision based Road Detection via On line Video Registration", Proceedings of the IEEE ITSC 2010.

[11] J. Chetan, K. Madhava and C. V. Jawahar, (2010), "An Adaptive Outdoor Terrain Classification Methodology using Monocular Camera", Proceedings of the IEEE IROS 2010.

[12] W. Yanqing, C. Deyun, S. Chaoxia and W. Peidong, (2010), "Vision-based Road Detection by Monte Carlo Method", Information Technology Journal, 9: 481-487.
[13] K. Yamaguchi, A. Watanabe, T. Naito and Y. Ninomiya, (2008), "Road region estimation using a sequence of monocular images", Proceedings of the International Conference on Pattern Recognition, 2008.

[14] N. Otsu, (1978), "A threshold selection method from graylevel histogram". IEEE Transactions on Systems, Man, and Cybernetics, vol. 9, pp. 62-66.

[15] M. Bertozzi. A. Broggi and A. Fascioli, (2000), "Vision-based intelligent vehicles: state of the art and perspectives", Robotics and Autonomous systems 32, 1-16.

[16] A. Miranda Neto, L. Rittner, N. Leite, D. E. Zampieri, R. Lotufo and A. Mendeleck, (2007), "Pearson's Correlation Coefficient for Discarding Redundant Information in Real Time Autonomous Navigation Systems", Proceedings of the IEEE MSC 2007.

[17] L. Benini, A. Bogliolo, and G. D. Micheli. (2000), "A Survey of Design Techniques for System-Level Dynamic Power Management", IEEE Transactions on Very Large Scale Integration Systems, 8(3):299-316.

[18] A. Miranda Neto, A. C. Victorino, I. Fantoni and D. E. Zampieri, (2011), "Real-Time Dynamic Power Management based on Pearson's Correlation Coefficient", Proceedings of the IEEE ICAR 2011.

[19] King Hann Lim et al., (2009), "Vision-based Lane-Vehicle Detection and Tracking", IAENG Transactions on Engineering Technologies Volume 3 - Special Edition', pp. 157-171, American Institute of Physics.

[20] S. Ettinger et al., (2003), "Vision-Guided Flight Stability and Control for Micro Air Vehicles", Adv. Robotics, 617-640.

[21] A. Miranda Neto, A. C. Victorino, I. Fantoni and D. E. Zampieri, (2011), "Robust Horizon Finding Algorithm for Real-Time Autonomous Navigation based on Monocular Vision", Proceedings of the IEEE ITSC 2011.

[22] A. Miranda Neto and L. Rittner, (2006), "A Simple and Efficient Road Detection Algorithm for Real Time Autonomous Navigation based on Monocular Vision", Proceedings of the IEEE 3rd LARS 2006.

[23] C. R. Gonzalez and E.R. Woods, (1991), "Digital Image Processing, Addison-Wesley Publishing Company.

[24] P. K. Sahoo, S. Soltani, and A. K. C. Wong, (1988), "A survey of thresholding techniques", Comput. Vision Graphics Image Processing, vol. 41, pp. 233-260.

[25] U.S. Lee, Y.S. Chung and H.R. Park, (1990), "A Comparative Performance Study of Several Global Thresholding Techniques for Segmentation". Computer Vision, Graphics, and Image Processing.

[26] M. Sezgin and B. Sankur, (2004), "Survey over image thresholding techniques and quantitative performance evaluation", Journal of Electronic Imaging; 13: 146-165.

[27] F. W. Rauskolb, et al., (2008), "Caroline: An autonomously driving vehicle for urban environments", Journal of Field Robotics, 25(9):674-724.

[28] J.F. Canny, (1986), "A computational approach to edge detection”. IEEE Trans. Pattern Anal. Machine Intell.

[29] D. Ballard, (1981), "Generalized Hough transform to detect arbitrary shapes", IEEE Trans. Pattern Anal. Machine Intell. 13 (2), 111-122.

[30] F. M. White, "Fluid Mechanics', 2nd Ed. McGraw Hill, 1986.

[31] DARPA 2005. "DARPA Grand Challenge".

[32] A. Miranda Neto, A. C. Victorino, I. Fantoni and D. E. Zampieri; J. V. Ferreira, (2012), "Visual-Perception Layer Applied to Reactive Navigation". Proceedings of the IEEE 9th LARS 2012.

[33] http://youtu.be/ZpEbRo32pY8 [retrieved Jan 31, 2013] 